\title{
Is it Possible to do Experimental Cosmology Using Cold Atoms?
}

\author{
Renato Ejnisman* and Nicholas P.Bigelow \\ Department of Physics and Astronomy and Laboratory for Laser Energetics \\ University of Rochester, Rochester NY 14627 USA \\ Received December 10, 1997
}

We analyze the possibility of using cold atoms to experimentally investigate the cosmic relic neutrinos and photons that were created after the Big Bang and presently constitute a background at temperatures of a few degrees kelvin.

\section{Introduction}

Since the discovery of the cosmic microwave background (CMB) by Penzias and Wilson [1], cosmology departed from the status of a philosophical discipline to that of a modern experimental branch of science. The CMB consists of photons at a temperature $T_{\gamma}=2.7 \mathrm{~K}$ that were originally created at about $10^{5}$ $\mathrm{s}$ after the Big Bang. More recent observations have shown an anisotropy in the background at the level $\Delta T_{\gamma} / T_{\gamma} \approx 10^{-5}$ that gives hints on how the process of galaxy formation took place [2].

An even more interesting and challenging achievement would be the detection of the relic cosmic neutrino background (CNB). The temperature of such neutrinos, $T_{\nu}=1.9 \mathrm{~K}$, implies a relatively sparse density ( $\rho_{\nu} \approx 300 \mathrm{~cm}^{-3}$ for zero chemical potential), which together with their extremely weak interaction with matter have eliminated any chances of direct observation up to date. Since the creation of the CNB occurred about 1s after the Big Bang, it serves as a probe to look that far back in the past. Its observation would therefore provide a very stringent test of the Big Bang theory and cosmological models, not to mention information about the physics of the neutrinos themselves.

The observation of the anisotropy of the CMB and especially the detection of the CNB comprise very difficult tasks, thus requiring extremely accurate experimental techniques. Recently, there have appeared in the literature many works and proposals of other precision experiments that make use of cold atoms ( $T \leq 100 \mu \mathrm{K})$. Such experiments and proposals entail a wide range of applications in physics with extremely high accuracy and relatively low cost. Examples are: atomic clocks (with expected precision of 1 part in $10^{16}$ ) [3], achievement of Bose-Einstein condensation [4], laser trapping of radioactive atoms $[5,6]$ and proposals for experiments to measure atomic parity violation [7], permanent electric dipole moments [8] and studies of $\beta$ decay [6]. Motivated by these results and proposals, we performed a thorough study to check the feasibility of employing cold atoms as a tool to observe the CMB and the $\mathrm{CNB}$, whose main results are reported in the present work. As will be shown, the conclusions for the CNB case are rather discouraging and for the CMB case quite tempting.

\section{Prospects for the CNB}

Since the typical cross-sections for interaction with neutrinos goes as $\sigma_{\nu} \approx G_{F}^{2} E_{\nu}^{2}$, one would obtain $\sigma_{\nu} \approx$ $10^{-62} \mathrm{~cm}^{2}$ for massless relic neutrinos or a factor of a few orders of magnitude higher for massive ones. Such a weak interaction deny any possibilities to detect the CNB by conventional methods.

In order to circumvent this problem, a number of experiments making use of coherent effects were proposed $[9,10,11]$. They rely on the fact that one can think of the neutrinos as de Broglie waves of wavelength

\footnotetext{
*e-mail: ejnisman@urhep.pas.rochester.edu
} 
much longer than the average size and spacing of the scatterers. However, Langacker et al [12] and Cabibbo and Maiani [13] showed that refs. [10] overestimated this effect. Also, Tupper et al [11] arrived at negative conclusions for their proposal. This leaves only the work of Stodolsky [9] as a possible (but improbable for the cases studied by him) route to measure the CNB. Other proposals to use resonant absorption of cosmic neutrinos [14] will not be discussed here (all proposals are reviewed in ref. [15]).

The idea of Opher and Lewis [10] relied on measurements of mechanical effects due to the total reflection of the neutrinos on a target of refraction index $n_{\nu}$ (for iron, e.g., assuming a density of $\left.10^{23} \mathrm{~cm}^{-3},\left|n_{\nu}-1\right| \cong 10^{-10}\right)$. Here forth, $\nu$ represents only the electron neutrino; the effects of the other neutrino flavors will be disregarded. Total reflection would occur for incidence angles below $\theta_{c}=\sqrt{2\left(1-n_{\nu}\right)}$. However, as shown in refs. [12, 13], there was a flaw in the calculation. The main problem was that the incident wave would have a penetration depth $D=\lambda / 2 \pi \theta_{c}$. This was by far larger than the size of the detectors considered, so most of the wave would actually be transmitted without transferring momentum to the target. Since $\left|n_{\nu}-1\right|$ goes linearly with density, things would be even worse for cold atoms. Typical densities of cold atomic samples are $\rho \approx 10^{10}-10^{13} \mathrm{~cm}^{-3}$, so $\left|n_{\nu}-1\right|$ would be smaller by at least a factor of about $10^{10}$ comparing to the case of iron.

Another scheme proposed by Stodolsky deserves a closer analysis. In his proposal [9], one would make use of the parity violating term of the $\mathrm{V}-\mathrm{A}$ Hamiltonian of the weak interactions. For a target consisting of a single electron, the term goes as $\vec{\sigma} \cdot \vec{v}$, where $\vec{\sigma}$ is the spin of the electron and $\vec{v}$ its velocity. Therefore, the two helicity states of the electron, corresponding to $\vec{\sigma} \cdot \vec{v}= \pm 1$, are split in energy. In fact, one can think of the neutrino background as having a velocity $\vec{v}$ with respect to an electron at rest on earth due to the motion of the solar system through the galaxy. For a target consisting of $\mathrm{N}$ particles, this would give rise to an energy shift between the case when the particles are polarized parallel or anti-parallel to the velocity $\vec{v}$ of [12]:

$$
\Delta E=\frac{4}{\sqrt{2}} G_{F} N v\langle\sigma\rangle\left(\rho_{\nu}-\rho_{\bar{\nu}}\right) \xi
$$

where $G_{F}$ is the weak coupling constant, $\langle\sigma\rangle$ is the aver- age spin of the target, $\rho_{\nu}\left(\rho_{\bar{\nu}}\right)$ is the number density of neutrinos (anti-neutrinos) and $\xi$ is a factor that equals 1 for massless neutrinos and $1 / 2$ for $m_{\nu} \gg k_{B} T_{\nu}$.

¿From the above, it is clear that this scheme would only yield non-zero results if there is an asymmetry between the number of neutrinos and anti-neutrinos. These particles should possess a Fermi distribution at a temperature $T_{\nu}=1.9 \mathrm{~K}$, such that the number density of neutrinos at energies corresponding to momenta between $q$ and $q+\delta q$ is given by [16]:

$$
\rho_{\nu}(q)=4 \pi h^{-3} q^{2} d q\left[\exp \left(\frac{q-\mu}{k_{B} T_{\nu}}\right)+1\right]^{-1}
$$

with an analogous expression for anti-neutrinos (with an opposite sign for the chemical potential $\mu$ ).

Although it is generally assumed that $\mu_{\nu}=0$, it is not possible to rule out a non-zero value for the chemical potential. In fact, in the case of complete degeneracy, $\left|\mu_{\nu}\right| \gg k_{B} T_{\nu}$, the density of neutrinos (in natural units) is given by:

$$
\rho_{\nu} \cong \frac{\mu_{\nu}^{3}}{6 \pi^{2}} ; \rho_{\bar{\nu}} \cong 0
$$

for the case of $\mu_{\nu}>0$. The case of negative $\mu_{\nu}$ swaps the role of neutrinos and anti- neutrinos.

The best current bounds on the chemical potentials come from indirect evidences. The fact that the neutrino energy should not be larger than the critical density, $\rho_{c}=2 \times 10^{-29} \mathrm{~g} \mathrm{~cm}^{-3}$, implies an upper limit on $\left|\mu_{\nu}\right|$ of about $6 \times 10^{-3} \mathrm{eV}$. Big Bang nucleosynthesis sets a more stringent limit of $-0.5 \leq \mu_{\nu} \leq 3$ in units of $10^{-4} \mathrm{eV}$ [17], which is however not as trustworthy as the bounds from the critical density [14]. Also of interest is the fact that if the sterile neutrino solution to the solar and atmospheric neutrino anomalies is true, then a non-zero value for $\mu_{\nu}$ is required to still make this solution compatible with Big Bang nucleosynthesis [18], which might prove to be a lower bound for $\left|\mu_{\nu}\right|$.

Assuming the quite unlikely case of complete degeneracy [19] and a target of $\mathrm{N}$ particles, the energy shift between spin parallel and anti-parallel to the earth's motion can be obtained by using (1) and (2):

$$
\Delta E \cong 6 \times 10^{-28} N\langle\sigma\rangle \mu_{\nu}^{3}
$$

where both $\Delta E$ and $\mu_{\nu}$ are in units of $\mathrm{eV}$. 
The cases analyzed by Stodolsky included a single trapped electron (for which $\Delta E \cong 6 \times 10^{-35} \mathrm{eV}$, using $\mu_{\nu}=6 \times 10^{-3} \mathrm{eV}$ ) and a large ferromagnet (for which $\Delta E \cong 6 \times 10^{-8} \mathrm{eV}$, for the same condition and $N=10^{27}$ ). In both cases, the numbers turn out to be very disappointing for any reasonable value of $\mu_{\nu}$.

One could think of using cold atoms in two ways. The first one is to take advantage of the extremely low temperatures that can be obtained with these atoms and try to verify a temperature difference between a sample polarized parallel or anti-parallel to the velocity of the solar system. In a recent experiment [20], about $10^{9}$ atoms of sodium of $\langle\sigma\rangle=1$ were initially captured in a magnetic trap and, by means of evaporative cooling, about $10^{7}$ atoms attained a temperature of about $1 \mu K$. Later, these atoms suffered adiabatic expansion which brought them to $T \approx 0.1 \mathrm{nK}$. Using $N=10^{7}$ and $\Delta E=9 \times 10^{-15} \mathrm{eV}$ (or $T \approx 10^{-10} \mathrm{~K}$ ), one could obtain an upper limit of $\left|\mu_{\nu}\right| \leq 100 \mathrm{eV}$. This bound is still rather weak when compared to the indirect limits mentioned above.

In a more optimistic scenario, one could use $\mathrm{Cs}^{1}$ which can be trapped at the $\mathrm{F}=4$ state (increasing the average spin) and can have about an order of magnitude more atoms than sodium captured in the magnetooptical trap (which is used as a first stage to load the magnetic trap [20]). Also, one could take advantage of the adiabatic expansion to decrease the temperature of the cloud even further. The temperature of an ideal gas in adiabatic expansion goes with the radius $R$ as $T \propto R^{-3(\gamma-1)}$. For a monoatomic gas, $\gamma=5 / 3$ and one obtains $T \propto R^{-2}$. In fact, this is an oversimplification of the problem because at these temperatures and densities collective effects should be taken into account. Even so, this simplified picture yields a temperature of $10^{-10} \mathrm{~K}$, which is close to the observed [20] for a cloud initially at $T=1 \mu \mathrm{K}$ and $R=10 \mu \mathrm{m}$ and final size of about $1 \mathrm{~mm}$. If we push this limit a little further, we can start with a cloud of radius $1 \mu \mathrm{m}$ and expand it to $1 \mathrm{~mm}$ to obtain a final temperature of $T=1 \mathrm{pK}$. For such temperatures, and assuming $N=10^{8}$ and $\langle\sigma\rangle=4$, it would be possible to obtain $\left|\mu_{\nu}\right| \leq 3 \mathrm{eV}$. Even though this limit is better than direct bounds obtained from measurements of $\beta$-decay spectra [21], indirect evidences still provide more stringent cut-offs for $\mu_{\nu}$. In fact, to achieve the limit $\left|\mu_{\nu}\right|<6 \times 10^{-3} \mathrm{eV}$, it would be necessary to have a sample with $N \cong 10^{16}$ atoms at a hypothetical temperature of $T=1 \mathrm{pK}$. It is hard to foresee the achievemet of such conditions in a near future, even though similar numbers were used in recent experimental proposals $[22,23]$. The requirements for each of the scenarios considered above are summarized in table 1.

Table 1 - Relevant numbers for the three scenarios studied and their estimated upper bound on the chemical potential $\mu$.

\begin{tabular}{||c|c|c|c||}
\hline \hline Scenario & Number of atoms & Temperature (K) & Upper limit on $\mu(\mathrm{eV})$ \\
\hline \hline Ref. 20 & $10^{7}$ & $10^{-10}$ & 100 \\
Optimistic & $10^{8}$ & $10^{-12}$ & 3 \\
Necessary & $10^{16}$ & $10^{-12}$ & $6 \times 10^{-3}$ \\
\hline \hline
\end{tabular}

An alternative route would be to try to measure the energy of the system directly. In atomic clocks, the transition $6 S_{1 / 2}(F=4) \rightarrow 6 S_{1 / 2}(F=5)$ of $\mathrm{Cs}$ is expected to yield results with a precision of $1 / 10^{16}$ using cold atoms [3]. This corresponds to an accuracy in en- ergy of $\Delta E \cong 4 \times 10^{-21} \mathrm{eV}$. Since this kind of measurement yields the energy of the transition of individual atoms, the energy shift from having the atoms polarized parallel or anti-parallel to the motion of the earth would not reflect that of the whole system. Therefore,

\footnotetext{
${ }^{1}$ On working with $\mathrm{Cs}$, one would have to deal with the issue of how to perform evaporative cooling, since Cs is believed to have a negative scattering length. Sympathetic cooling with another element of positive scattering length might be a way to circumvent this drawback.
} 
eq. (1) would not contain the factor $N$ in this case. Hence, one would obtain the weak bound $\left|\mu_{\nu}\right|<120$ $\mathrm{eV}$.

\section{Prospects for the CMB}

In the case of the cosmic microwave background, one has to check not only for the feasibility of schemes for observation, but also for how they compare to existing methods. A possible way to use cold atoms to detect the CMB can be the following: start with a sample of atoms in a magnetic trap with all the atoms in the lower hyperfine state (e.g., $\mathrm{F}=4$ for $\mathrm{Cs}$ ); then, photons from the CMB with energies close to that of the $6 S_{1 / 2}(F=4) \rightarrow 6 S_{1 / 2}(F=3)$ transition $(\mathrm{f}=9.2 \mathrm{GHz})$ could promote some of the trapped atoms to the lower untrapped state which would create a loss mechanism for such traps.

The loss rate from the trap could be measured with the trap shielded from the CMB and then compared with the unshielded case. From the difference in loss rate, the contribution due to loss from CMB absorption could be inferred. A somewhat similar experiment was performed by Schappe and coworkers to measure the cross-section for collisions between electrons and atoms [24]. In our case, instead of the cross-section, one would be interested in obtaining the flux of photons and, from that, the temperature of this background radiation.

The work with electrons yielded cross-sections with uncertainties of about 10\% [24]. Assuming that for the case of CMB the uncertainty on the photon flux obtained by this method is of $10 \%$, one would obtain an uncertainty in the temperature of $7 \%$. This is to be compared with observations of the CMB at comparable wavelengths that have a precision on absolute temperatures of about $2 \%$ [25]. Optimized traps should be able to yield results of such precision for reasonable loss rates.

Before we move on to obtain the loss rate of atoms due to the interaction with the CMB, it is important to consider a technical difficulty that would have to be faced. It comes from the fact that at $9.2 \mathrm{GHz}$, the number of photons from the low energy tail of the blackbody radiation at room temperature is about two orders of magnitude higher than the one due to the CMB. A way around that is to use ${ }^{4} \mathrm{He}$ cryostats that can cool the system down to about $2 \mathrm{~K}$ at the lowest temperatures achievable [26], which is sufficiently low for the blackbody radiation due to ambient temperatures not to pose major difficulties.

The cross-section for a $F=4 \rightarrow F=3$ transition can be calculated by standard equations [27]. From them, a CMB photon flux of approximately $20 \mathrm{~cm}^{-2} \mathrm{~s}^{-1} \mathrm{~Hz}^{-1}$ and a $10^{4}$ enhancement due to the use of a horn with an area of the order of $1 \mathrm{~m}^{2}$, one obtains a loss rate of approximately $10^{4}$ atoms per second for a trap of $10^{10}$ atoms. This is certainly unacceptable compared to a typical lifetime of about one minute for these traps [20].

A way to improve this scenario is to employ high$\mathrm{Q}$ cavities to increase the absorption of photons and therefore the loss rate due to the interaction with the CMB. Cavities of $Q$ up to $10^{6}$ have been used with magnetic fields inside for experiments with Rydberg atoms [26]. The loss rate would then be increased by a factor $Q \lambda^{3} / V$, where $\lambda \approx 3 \mathrm{~cm}$ for $9.2 \mathrm{GHz}$ and $V$ is the cavity mode volume. If the loss rate is increased in this way by $10^{5}$, it will become approximately $10^{9} \mathrm{~s}^{-1}$ for a trap with $10^{10}$ atoms, which is now a measurable rate. As stated before, the challenge then would be to make sure that a $2 \%$ precision is achieved as in the current existing methods.

It is important to notice that a nice feature of this kind of measurement is that the "antena" we use (i.e., the atoms) have a narrow band for absorption. This might be interesting for studies of the CMB that envolve detailed knowledge of the microwave frequencies. Another good facet of the present technique is that the part of the CMB spectrum where it would enable investigations (low energy tail of the blackbody spectrum) is more subject to eventual distortions of a Plack distribution [25].

Of course, other ways can be thought of to approach this problem. For example, one could perform experiments with superconducting cavities (of $Q \approx 10^{9}-$ $10^{11}$ ) in a microgravity environment where the atoms are initially prepared in a certain state (e.g., by optical pumping). A subsequent observation would reveal if they had absorbed photons and changed to another state. In fact, for this case, the use of cold Rydberg atoms would be even more appropriate since their large dipole moments enable them to interact more strongly 
with microwave radiation. Even though the interaction of blackbody radiation with Rydberg atoms has been observed for temperatures down to $\mathrm{T}=50 \mathrm{~K}[26,28]$, it is not clear how it would be possible to obtain a precision of $2 \%$ at the even lower temperatures of the CMB. Also, the fact that a microgravity environment is wanted would make this experiment much more costly.

\section{Conclusions}

We have analyzed the possibility to use cold atoms as a tool to detect the cosmic neutrino background and the cosmic microwave background. In the former case, although it would be possible to obtain upper limits on the neutrino chemical potential, these limits would not be competitive with other bounds estimated through indirect evidences. In the latter case, the method considered to measure the flux of photons from the CMB may be able to yield results with levels of precision close to that of other existing methods and should therefore be further explored.

To conclude, we would like to state that we are confident that techniques similar to the ones considered here and presented in the references of the introduction are a good alternative to make precise and fundamental physics at relatively low costs. In particular, we believe that analyses such as the one performed in this work will eventually lead to a feasible way to perform the important task of detecting the cosmic neutrino background.

We are very grateful to A.C.Melissinos for essential discussions. R.E. was supported by a CNPq fellowship (Brazil). This work was supported by the NSF and the David and Lucile Packard Foundation.

\section{References}

[1] A. A. Penzias and E. W. Wilson, Ap. J. 142, 419 (1965).

[2] G. Smoot et al, Ap. J. 396, L1 (1992).
[3] A. Clairon et al, Europhys. Lett. 16, 165 (1991); K. Gibble and S. Chu, Phys. Rev. Lett. 70, 1771 (1993).

[4] M. H. Anderson et al, Science 269, 198 (1995); K. B. Davis et al, Phys. Rev. Lett. 75, 3969 (1995).

[5] J. E. Simsarian et al, Phys. Rev. Lett. 76, 3522 (1996); G. Gwinner et al, Phys. Rev. Lett. 72, 3795 (1994).

[6] Z-T. Lu et al, Phys. Rev. Lett. 72, 3791 (1994).

[7] C. E. Wieman, Hyp. Int. 81, 27 (1993).

[8] M. Bijlsma et al, Phys. Rev. A49, R4285 (1994).

[9] L. Stodolsky, Phys. Rev. Lett. 34, 110 (1974).

[10] R. Opher, Astron. Astrophys. 37, 135 (1974); R. R. Lewis, Phys. Rev. D21, 663 (1980).

[11] G. Tupper et al, Phys. Rev. D35, 394 (1987).

[12] P. Langacker et al, Phys. Rev. D27, 1128 (1983).

[13] N. Cabibbo and L. Maiani, Phys. Lett. B114, 115 (1982).

[14] T. Weiler, Phys. Rev.Lett. 49, 234 (1982).

[15] B. Müller, in Neutrino Physics, H. V. Klapdor and B. Povh (eds.), (Springer- Verlag, New York, 1988), p. 269

[16] S. Weinberg, Gravitation and Cosmology, (John Wiley, New York, 1972)

[17] G. Beaudet and P. Goret, Astron. Astrphys. 49, 415 (1976).

[18] R. Foot and R. R. Volkas, Phys. Rev. Lett. 75, 4350 (1995).

[19] B. A. Campbell and P. J. O’Donnell, Phys. Rev. D26, 1487 (1982)

[20] M.-O. Mewes et al, Phys. Rev. Lett. 77, 416 (1996).

[21] Particle Data Group, Phys. Rev. D50, 1173 (1994).

[22] J. J. Hope and C. M. Savage, Phys. Lett. A222, 87 (1990).

[23] V. A. Namiot and V. I. Goldanskii, Phys. Lett. A213, (1996).

[24] R. S. Schappe et al, Phys. Rev. Lett. 76, 4328 (1996).

[25] M. Bersanelli et al, in Present and Future of Microwave Background, J.L.Sanz et al (eds.), (Springer-Verlag , New York, 1994), p.1.

[26] S. Haroche and J. M. Raimond, Adv. Atom. Mol. Phys., 20, 347 (1985).

[27] C. H. Townes and A. L. Schawlow, Microwave Spectroscopy, (McGraw-Hill, New York, 1955), p. 146.

[28] It should be noted that an experiment where cold atoms were trapped in a cryogenic environment has already been realized. See: P.A. Willems and K.G. Libbrecht, Phys. Rev. A51, 1403 (1995). 